\title{
Impact of national policy initiatives on fatal and non-fatal self-harm after psychiatric hospital discharge: time series analysis ${ }^{\dagger}$
}

David Gunnell, Chris Metcalfe, David While, Keith Hawton, Davidson Ho, Louis Appleby and Nav Kapur

\section{Background}

Risk of self-harm and suicide is greatly increased in the period after discharge from psychiatric in-patient care.

\section{Aims \\ To investigate the impact on suicide of a series of policy initiatives to enhance care in the immediate post-discharge period. \\ Method \\ A time series analysis was based on 1997-2007 data from the National Confidential Inquiry into Suicide and from Hospital Episode Statistics for England.}

\section{Results}

There was no evidence of a reduced risk of suicide in the first 12 weeks following discharge in 2003-2007 compared with 1997-2002. In contrast, the relative risk of non-fatal selfharm in the 12 weeks after discharge declined. The risk ratio for self-harm (2003-2007 v. 1997-2002) at 0-1 week postdischarge was $0.86(95 \% \mathrm{Cl} 0.80-0.92)$ and at $2-4$ weeks it was 0.89 (95\% Cl 0.85-0.94).

\section{Conclusions}

These findings provide some support for the impact of recent policy changes on the risk of non-fatal self-harm in the immediate period after discharge from psychiatric in-patient care.

\section{Declaration of interest}

None.
The first few weeks after discharge from psychiatric hospital in-patient care are a period of extremely high risk of self-harm and suicide. Studies in the UK, Denmark, Singapore and Hong Kong show that the risk of suicide is up to 200 times higher than that of the general population around this time. ${ }^{1-6}$ Risk declines rapidly in the subsequent weeks before reaching a relatively steady state at around 6 months after discharge. In England approximately $5 \%$ of all suicides (220 per year) occur during the 3 months after discharge from psychiatric hospital. ${ }^{2}$ In keeping with this pattern, recent data indicate that the risk of non-fatal self-harm is also greatly increased in the post-discharge period: within 12 months of discharge, over $6 \%$ of all psychiatric hospital patients were admitted to a general hospital following an episode of self-harm. ${ }^{7}$

Since 2002 a number of policy initiatives aimed at reducing suicide risk in the post-discharge period have been introduced in England. A specific action recommended in the National Suicide Prevention Strategy for England (September 2002) was that following psychiatric hospital discharge, everyone with severe mental illness or a history of self-harm in the previous 3 months should be followed up in the community within 7 days. ${ }^{8}$ The Department of Health's guidance for mental health services in 2003 recommended that in-patient and community teams carry out a joint case review prior to discharge; that care plans should take into account the heightened risk of suicide in the first 3 months after discharge and make specific reference to the first week; and that patients who were thought to be at high risk of suicide during their admission should be followed up within $48 \mathrm{~h}$ of discharge. ${ }^{9}$ To date, the impact of these initiatives on the risk of fatal and non-fatal self-harm in the early weeks following hospital discharge has not been assessed. In this study we use data collected in the National Confidential Inquiry into Suicide and Homicide

'See editorial, pp. 175-177, this issue. by People with Mental Illness, ${ }^{10}$ and from Hospital Episode Statistics, to address the hypothesis that policy interventions have reduced fatal and non-fatal self-harm in the early weeks after psychiatric hospital discharge.

\section{Method}

\section{Data sources}

National Confidential Inquiry data for England 1997-2007

Data collection for the National Confidential Inquiry occurs in three stages. First, information on all deaths among those aged 15-95 years in England receiving a suicide or open (undetermined) verdict at a coroner's inquest is obtained from the Office for National Statistics. Second, information on whether the person had been in contact with secondary mental health services in the 12 months before death is obtained from National Health Service (NHS) trusts. Third, clinical data about people who had been in contact with services (referred to as 'Inquiry cases') are obtained by sending a questionnaire to the consultant psychiatrist who had been in charge of their care. This questionnaire included patient status (in-patient, community patient) and timing of death. Further details of Inquiry methodology are available elsewhere. ${ }^{10}$ Case ascertainment and completion rates for questionnaires are consistently high $(>95 \%){ }^{10}$

\section{Hospital Episode Statistics data for England 1997-2007}

Hospital Episode Statistics (HES) data record patients admitted to NHS hospitals in England; admissions to private hospitals are not included. Using approaches developed in previous analyses of HES psychiatric admission data, ${ }^{7}$ we selected all patients aged 16-64 years who had been discharged from hospital following an episode of care under a psychiatric specialist (HES main specialty code 
710: mental illness) on at least one occasion during the 11-year study period. This code excludes the relatively few patients admitted under the following psychiatric specialties: learning disability, child and adolescent psychiatry, forensic psychiatry and psychotherapy.

We created unique patient identifiers, derived from the person's home postcode, date of birth and gender, to enable us to link the admissions of each individual patient. Using that identifier, and based on each patient's first episode of psychiatric in-patient care in each study year, we searched the HES databases for the 12 months following discharge to identify hospital readmissions for self-harm. Any readmission occurring on the same day as the discharge date was removed from the analysis to ensure we excluded patient transfers. We used the following ICD-10 codes to identify admissions for self-harm: X60-X84 (intentional self-harm), Y10-Y34 (event of undetermined intent) and X40-49 (accidental poisoning by exposure to noxious substances). ${ }^{11}$ The latter codes were used because most such episodes are probable acts of self-harm: the sociodemographic characteristics of people with these diagnoses are similar to those of people admitted following self-harm, and accidental poisoning in the 16-64 year age group is unusual. Previous studies have found that Hospital Episode Statistics record hospital admission following self-poisoning reasonably reliably. ${ }^{12}$

\section{Statistical analysis}

We examined the proportion of people dying by suicide or admitted following self-harm in the 12 months following psychiatric hospital discharge who did so in week 1, weeks 2-4 and weeks 5-12 of that period. Comparing these proportions across calendar years, we looked for evidence of an effect of policy initiatives on early post-discharge fatal and non-fatal self-harm. By considering people dying by suicide or admitted to hospital following self-harm in the 12 months following discharge as the population at risk of fatal or non-fatal self-harm early in that period, secular changes in psychiatric hospital case mix and the background rate of suicide or self-harm are partly accommodated. Nevertheless, to assess the possible impact of changes in case mix on the associations we observed, we conducted a secondary analysis controlling for the primary diagnosis at discharge - eight slightly different diagnostic categories such as depression, psychosis and substance misuse were recorded for the cases in the two data-sets.

We use the log-rank test to compare the equality of survival functions in 1997-2002 v. 2003-2007 across the critical first 12 weeks after discharge. Poisson regression models were used to estimate the change across the study period in the risk of fatal or non-fatal self-harm in the early weeks after psychiatric hospital discharge. The risk in a particular period was calculated as the number of events in that period divided by the number of people who had not yet experienced an event at the beginning of that period. For example, the risk of an event during the 2-4 week period was calculated as the number of events in that period divided by the number of people who had not already experienced an event during the $0-1$ week period. Evidence of a linear trend in these risks across the study period was evaluated by including year as a covariate in the regression model. The risks before (and including) 2002 - the year before policy changes regarding postdischarge psychiatric care were introduced - were compared with

\begin{tabular}{|c|c|c|c|c|c|}
\hline & $\begin{array}{c}\text { 0-1 week } \\
n(\%)\end{array}$ & $\begin{array}{c}\text { 2-4 weeks } \\
n(\%)\end{array}$ & $\begin{array}{c}\text { 5-12 weeks } \\
n(\%)\end{array}$ & $\begin{array}{c}\text { 13-52 weeks } \\
n(\%)\end{array}$ & $\begin{array}{c}\text { Total: } 0-52 \text { weeks } \\
n\end{array}$ \\
\hline \multicolumn{6}{|l|}{ Suicide $^{a}$} \\
\hline 1997 & $31(6)$ & 89 (19) & $126(26)$ & $231(48)$ & 477 \\
\hline 1998 & $42(8)$ & $94(19)$ & $116(23)$ & $256(50)$ & 508 \\
\hline 1999 & $43(9)$ & 89 (19) & $115(25)$ & $218(47)$ & 465 \\
\hline 2000 & $36(7)$ & $89(17)$ & $144(27)$ & $256(49)$ & 525 \\
\hline 2001 & $33(7)$ & $93(19)$ & $138(28)$ & $237(47)$ & 501 \\
\hline 2002 & $50(10)$ & $68(14)$ & $116(23)$ & $260(53)$ & 494 \\
\hline Summary of 1997-2002 & $235(8)$ & $522(18)$ & 755 (25) & $1458(49)$ & 2970 \\
\hline 2003 & $36(7)$ & $76(16)$ & $125(26)$ & $249(51)$ & 486 \\
\hline 2004 & $39(8)$ & 79 (17) & $104(22)$ & $248(53)$ & 470 \\
\hline 2005 & $32(7)$ & $71(16)$ & $102(24)$ & $226(52)$ & 431 \\
\hline 2006 & $28(7)$ & $57(15)$ & $108(29)$ & 185 (49) & 378 \\
\hline 2007 & $35(9)$ & $72(19)$ & $85(22)$ & $189(50)$ & 381 \\
\hline Summary of 2003-2007 & $170(8)$ & $355(17)$ & $524(24)$ & $1097(51)$ & 2146 \\
\hline Total & $405(8)$ & 877 (17) & $1279(25)$ & $2555(50)$ & 5116 \\
\hline \multicolumn{6}{|l|}{ Self-harm admissions ${ }^{b}$} \\
\hline 1997 & $398(9)$ & $663(15)$ & $993(22)$ & $2389(54)$ & 4443 \\
\hline 1998 & $328(8)$ & $603(15)$ & $910(22)$ & $2257(55)$ & 4098 \\
\hline 1999 & $327(8)$ & $543(14)$ & $889(23)$ & $2178(55)$ & 3937 \\
\hline 2000 & $325(9)$ & 519 (14) & $822(22)$ & $2131(56)$ & 3797 \\
\hline 2001 & $310(9)$ & $513(15)$ & $678(19)$ & $2003(57)$ & 3504 \\
\hline 2002 & $317(9)$ & 447 (12) & $734(20)$ & 2149 (59) & 3647 \\
\hline Summary of 1997-2002 & 2005 (9) & 3288 (14) & $5026(21)$ & $13107(56)$ & 23426 \\
\hline 2003 & $306(8)$ & $483(12)$ & $834(21)$ & $2326(59)$ & 3949 \\
\hline 2004 & $289(7)$ & $561(14)$ & $856(21)$ & 2448 (59) & 4154 \\
\hline 2005 & $264(6)$ & $529(13)$ & $848(21)$ & $2440(60)$ & 4081 \\
\hline 2006 & $283(7)$ & 503 (13) & $802(21)$ & $2197(58)$ & 3785 \\
\hline 2007 & $291(8)$ & $428(11)$ & $775(20)$ & $2297(61)$ & 3791 \\
\hline Summary of 2003-2007 & $1433(7)$ & 2504 (13) & $4115(21)$ & 11708 (59) & 19760 \\
\hline Total & 3438 (8) & 5792 (13) & 9141 (21) & 24815 (57) & 43186 \\
\hline
\end{tabular}


those in 2003-2007 by including a dummy variable in the models (0, 1997-2002; 1, 2003-2007). All models included a dummy variable distinguishing male and female patients. We investigated whether any changes in risk of fatal or non-fatal self-harm risk over time differed between men and women and between younger $v$. older patients (using median age in each data-set as the cut-off point) by including interaction terms.

\section{Results}

Over the 11-year study period there were 5116 suicides and 43186 hospital admissions for self-harm within 12 months of discharge from psychiatric in-patient care. The 5116 suicides represented $10 \%$ of the 51596 suicides that occurred in England over this period. Table 1 shows, by year, the timing of suicides and selfharm admissions over the 12 months following the index discharge from psychiatric in-patient care. Half of all suicides (50\%) and $43 \%$ admissions for self-harm in the 12 months following discharge occurred within the first 12 weeks of that period, and $25 \%$ and $21 \%$ respectively occurred within the first 4 weeks. There were declines in the numbers of cases of fatal and non-fatal self-harm over the study period, reflecting a decline in the number of discharges from psychiatric in-patient units from 90831 in 1997 to 64033 in 2007. There was no clear evidence of substantial reductions in the proportion of suicides or hospital admissions for self-harm in the first few weeks following hospital discharge, although the absolute number of events declined over the study period (in keeping with declines in in-patient admissions).

Figure 1 shows the cumulative risk of (a) suicide and (b) hospital admission for self-harm in the first 12 weeks following psychiatric hospital discharge for the periods before and after the policy change. There is some (slight) separation of the lines in each graph, indicating a possible beneficial impact of changes in post-discharge policy, although statistical evidence of this difference was only apparent in relation to hospital admission for self-harm - log-rank test values for the equality of survival functions in 1997-2002 v. 2003-2007 were $\chi^{2}=0.16$, d.f. $=2$, $P=0.69$ for suicide and $\chi^{2}=52.86$, d.f. $=2, P<0.001$ for hospital admission for self-harm.

\section{Poisson regression models}

For suicide (Table 2) there was no evidence of a year-on-year decline in suicide risk across the study period for any of the three

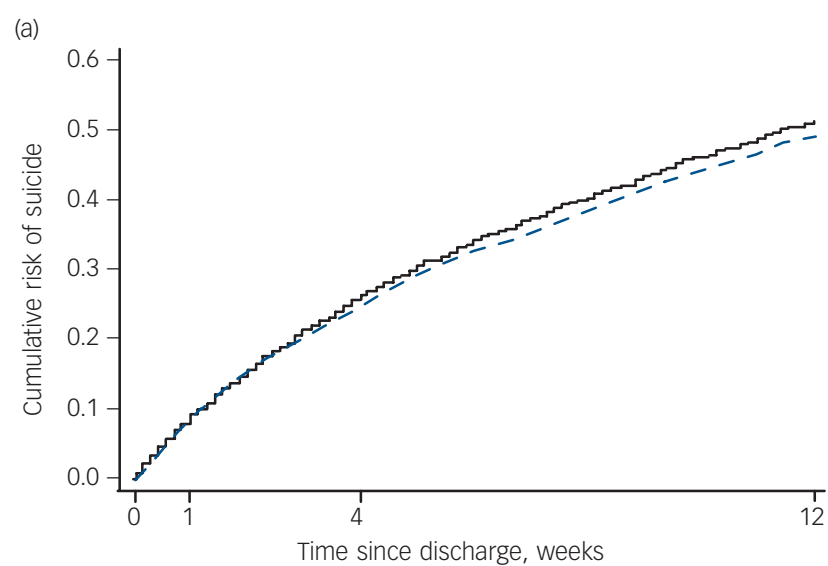

(b)

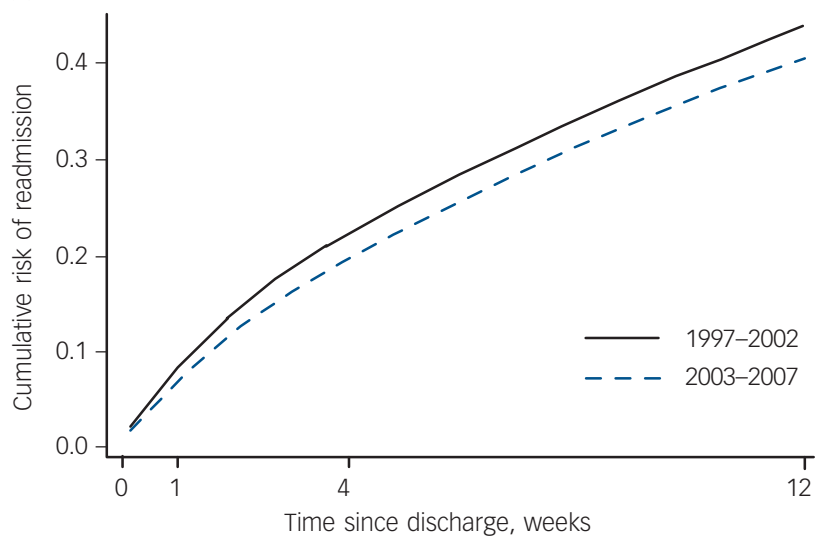

Fig. 1 Cumulative risk in England 1997-2007 of people who (a) died by suicide, age 15-95 years, or (b) were admitted to hospital following self-harm, age 16-64 years, following discharge from psychiatric hospital in-patient care.

immediate post-discharge periods (risk ratios: 0-1 week, $\mathrm{RR}=1.01 ; 2-4$ weeks, $\mathrm{RR}=0.99 ; 5-12$ weeks, $\mathrm{RR}=0.99$ ). Looking at the year-specific rate ratios around the year 2003, there was no suggestion of a fall in the rates of suicide in the weeks immediately post-discharge that might be attributed to the initiatives introduced at that point. There was no convincing

Table 2 Change over the study period (1997-2007) in the gender-adjusted risk of suicide within the specified time from the index psychiatric in-patient admission, among male and female residents of England, aged 15-95 years, who died following self-harm within 1 year of discharge

\begin{tabular}{|c|c|c|c|}
\hline Year & $\begin{array}{c}\text { 0-1 week } \\
\text { RR }(95 \% \mathrm{Cl})\end{array}$ & $\begin{array}{l}2-4 \text { weeks } \\
\text { RR }(95 \% \mathrm{Cl})\end{array}$ & $\begin{array}{l}\text { 5-12 weeks } \\
\text { RR (95\% Cl) }\end{array}$ \\
\hline 1997 & $0.64(0.41-1.00)$ & $1.30(0.95-1.79)$ & $1.14(0.89-1.47)$ \\
\hline 1998 & $0.82(0.54-1.23)$ & $1.32(0.96-1.80)$ & $1.01(0.78-1.31)$ \\
\hline 1999 & $0.91(0.61-1.37)$ & $1.38(1.01-1.89)$ & $1.12(0.86-1.45)$ \\
\hline 2000 & $0.68(0.44-1.04)$ & $1.19(0.86-1.63)$ & $1.17(0.92-1.49)$ \\
\hline 2001 & $0.65(0.42-1.01)$ & $1.30(0.95-1.77)$ & $1.19(0.93-1.53)$ \\
\hline 2002 & 1.00 & 1.00 & 1.00 \\
\hline 2003 & $0.73(0.48-1.13)$ & $1.10(0.79-1.53)$ & $1.09(0.84-1.40)$ \\
\hline 2004 & $0.82(0.54-1.25)$ & $1.20(0.86-1.65)$ & $0.96(0.74-1.25)$ \\
\hline 2005 & $0.74(0.47-1.15)$ & $1.16(0.83-1.61)$ & $1.01(0.77-1.32)$ \\
\hline 2006 & $0.73(0.46-1.16)$ & $1.06(0.75-1.51)$ & $1.20(0.92-1.55)$ \\
\hline 2007 & $0.91(0.59-1.40)$ & $1.36(0.98-1.89)$ & $1.01(0.76-1.33)$ \\
\hline Year-on-year trend & $\begin{array}{c}1.01(0.98-1.04) \\
\chi^{2}=0.38, \text { d.f. }=1, P=0.54\end{array}$ & $\begin{array}{c}0.99(0.97-1.01) \\
\chi^{2}=1.41, \text { d.f. }=1, P=0.23\end{array}$ & $\begin{array}{c}0.99(0.98-1.01) \\
\chi^{2}=0.63, \text { d.f. }=1, P=0.43\end{array}$ \\
\hline $\begin{array}{l}\text { Risk in 2003-2007 compared } \\
\text { with 1997-2002 }\end{array}$ & $\begin{array}{c}1.00(0.82-1.22) \\
\chi^{2}<0.01, \text { d.f. }=1, P=0.98\end{array}$ & $\begin{array}{c}0.94(0.82-1.08) \\
\chi^{2}=0.81, \text { d.f. }=1, P=0.37\end{array}$ & $\begin{array}{c}0.95(0.85-1.06) \\
\chi^{2}=0.88, \text { d.f. }=1, P=0.35\end{array}$ \\
\hline
\end{tabular}


evidence that the risk of suicide was lower in the weeks immediately following discharge when comparing 2003-2007 with 1997-2002. During 2003-2007 there was no evidence of a reduced risk in the post-discharge period, although all risk ratios were 1.0 or below, with the lowest risk seen in weeks $2-4(\mathrm{RR}=0.94,95 \%$ CI $0.82-1.08 ; P=0.37$ ); however, reductions of this magnitude are consistent with chance. There was no evidence that these effects differed in male $v$. female patients $(P>0.20$ for interaction) or in younger $v$. older patients $(P>0.80$ for interaction). In a series of sensitivity analyses we used different years (2003 and 2004) as cut-off points for the hypothetical impact of post-discharge interventions, but our conclusions were unchanged. There was little evidence that the main psychiatric diagnoses contributing to suicide after hospital discharge changed over the 11-year study period. In the 1997-2002 period $18 \%$ of suicide cases had a primary diagnosis of schizophrenia and $31 \%$ had depression; in 2003-2007 the equivalent figures were 18\% and 33\% respectively. Controlling for psychiatric diagnosis had little impact on the risk ratios, e.g. for the $2-4$ weeks following discharge the risk ratio decreased slightly from 0.94 to 0.93 (95\% CI $0.81-1.07$ ).

Poisson regression models for hospital admission for selfharm are shown in Table 3. There was strong evidence of a year-on-year decline in risk of hospital admission for self-harm in the weeks following discharge from in-patient psychiatric care (change in risk per year: week $0-1, R R=0.98$; weeks $2-4$, $\mathrm{RR}=0.98$; weeks $5-12, \mathrm{RR}=0.99$; all $P<0.001$ ). Inspecting the year-specific risk ratios, there is a suggestion that the decline in the risk of admission due to self-harm in the week following discharge from psychiatric in-patient care occurred around 2003, when the initiatives were introduced. However, for the 2-4 week and 5-12 week post-discharge periods, the reduction in the risk of self-harm may have preceded the initiatives. These reductions in the risk of early post-discharge self-harm were also clearly observed when the period 2003-2007 was compared with 1997-2002 (0-1 week, $\mathrm{RR}=0.86$, 95\% CI 0.80-0.92; 2-4 weeks, $\mathrm{RR}=0.89,95 \%$ CI $0.85-0.94 ; 5-12$ weeks, $\mathrm{RR}=0.94,95 \%$ CI $0.90-0.98)$. There was no strong evidence that these effects differed in men and women (interaction tests: $P=0.081$ for the $0-1$ week post-discharge period, where there was a suggestion of a greater reduction in self-harm among male patients; $P=0.73$ for the $2-4$ week period; $P=0.53$ for the $5-12$ week period). There was some evidence that that the effects were stronger in younger than in older patients (interaction test probability values at $0-1$ week, 2-4 weeks and 5-12 weeks post-discharge were $P=0.64$, $P=0.011$ and $P=0.17$ respectively); at $2-4$ weeks the risk ratio was $0.85(95 \%$ CI $0.79-0.91)$ in the younger group and 0.97 (95\% CI 0.90-1.04) in the older group.

As seen with the analysis of the National Confidential Inquiry data there was little evidence that the main psychiatric diagnoses contributing to self-harm after hospital discharge changed over the study period. In the $1997-2002$ period, $8 \%$ of self-harm cases had a primary diagnosis of schizophrenia and $34 \%$ had a diagnosis of depression; in 2003-2007 the equivalent figures were 9\% and $31 \%$ respectively. Controlling for psychiatric diagnosis had little impact on the risk ratios, for example for the 2-4 weeks following discharge the risk ratio increased slightly from 0.89 to 0.90 (95\% CI 0.85-0.95). Our conclusions were unchanged in sensitivity analyses when we used different years (2003 and 2004) as the cut-off points to assess the impact of policy changes.

\section{Discussion}

We found no evidence that policy changes designed to reduce the risk of post-discharge suicide resulted in a reduction in deaths, although the confidence interval around the relative risk of suicide at 2-4 weeks following discharge $(0.94,95 \%$ CI $0.82-1.08)$ does not rule out an $18 \%$ reduction in risk (or an $8 \%$ increase). For self-harm admissions we observed a $14 \%$ (95\% CI $8-20)$ reduction in risk in the first week following discharge in 2003-2007 compared with 1997-2002 and a slightly smaller reduction in later weeks. This finding must be interpreted in the light of multiple hypothesis testing - our primary analyses were based on six different post-discharge period (0-1 week, 2-4 weeks, 5-12 weeks) and outcome (fatal/non-fatal self-harm) combinations.

\section{Strengths and limitations}

We used national suicide and attempted suicide data to assess the impact of policy changes on suicide. To the best of our knowledge, this is the largest evaluation of the impact of mental health policy surrounding post-discharge aftercare on suicide risk carried out to date.

There are a number of limitations to this analysis. First, we have assumed that policy recommendations were widely implemented,

Table 3 Change over the study period (1997-2007) in the gender-adjusted risk of self-harm related admission within the specified time following the index psychiatric in-patient episode, among male and female residents of England, aged 16-64 years, who were admitted following self-harm within 1 year of discharge

\begin{tabular}{|c|c|c|c|}
\hline Year & $\begin{array}{l}\text { 0-1 week } \\
\text { RR }(95 \% \mathrm{Cl})\end{array}$ & $\begin{array}{l}\text { 2-4 weeks } \\
\text { RR }(95 \% \mathrm{Cl})\end{array}$ & $\begin{array}{l}\text { 5-12 weeks } \\
\text { RR }(95 \% \mathrm{Cl})\end{array}$ \\
\hline 1997 & $1.02(0.88-1.18)$ & $1.21(1.08-1.37)$ & $1.15(1.05-1.26)$ \\
\hline 1998 & $0.91(0.78-1.06)$ & 1.19 (1.05-1.34) & $1.13(1.02-1.24)$ \\
\hline 1999 & $0.95(0.81-1.10)$ & $1.12(0.98-1.26)$ & $1.14(1.03-1.25)$ \\
\hline 2000 & $0.98(0.84-1.14)$ & $1.11(0.98-1.26)$ & $1.09(0.99-1.21)$ \\
\hline 2001 & $1.02(0.87-1.19)$ & $1.20(1.05-1.36)$ & $0.99(0.90-1.10)$ \\
\hline 2002 & 1.00 & 1.00 & 1.00 \\
\hline 2003 & $0.89(0.76-1.04)$ & $0.99(0.87-1.12)$ & $1.04(0.94-1.14)$ \\
\hline 2004 & $0.80(0.68-0.94)$ & $1.08(0.96-1.23)$ & $1.02(0.92-1.12)$ \\
\hline 2005 & $0.75(0.63-0.88)$ & $1.03(0.91-1.17)$ & $1.01(0.92-1.12)$ \\
\hline 2006 & $0.86(0.73-1.01)$ & $1.07(0.94-1.22)$ & $1.05(0.95-1.16)$ \\
\hline 2007 & $0.89(0.76-1.04)$ & $0.91(0.80-1.04)$ & $0.99(0.90-1.10)$ \\
\hline Year-on-year trend & $\begin{array}{c}0.98(0.97-0.99) \\
\chi^{2}=13.3, \text { d.f. }=1, P<0.001\end{array}$ & $\begin{array}{c}0.98(0.97-0.99) \\
\chi^{2}=25.7, \text { d.f. }=1, P<0.001\end{array}$ & $\begin{array}{c}0.99(0.98-0.99) \\
\chi^{2}=17.4, \text { d.f. }=1, P<0.001\end{array}$ \\
\hline Risk in 2003-2007 compared with 1997-2002 & $\begin{array}{c}0.86(0.80-0.92) \\
\chi^{2}=20.6, \text { d.f. }=1, P<0.001\end{array}$ & $\begin{array}{c}0.89(0.85-0.94) \\
\chi^{2}=18.1, \text { d.f. }=1, P<0.001\end{array}$ & $\begin{array}{c}0.94(0.90-0.98) \\
\chi^{2}=8.5, \text { d.f. }=1, P=0.004\end{array}$ \\
\hline
\end{tabular}


but we did not collect data on implementation as part of this study. However, the National Confidential Inquiry does collect self-completed data from NHS trusts which suggest that policies on follow-up within 7 days of discharge are among the most widely implemented of all Inquiry recommendations. ${ }^{10}$ Second, by using fatal and non-fatal self-harm occurring in weeks $12-52$ following discharge as our reference category, we have assumed that none of the would-be suicides possibly prevented in the early weeks following discharge were simply postponed to the later period of follow-up. If this were to occur it would exaggerate the real impact of recent policy changes. Some reassurance can be taken from the observation that reductions in fatal and nonfatal self-harm in the first week were not accompanied by increases in the subsequent weeks, although such an effect could be obscured by the overall reduction in rates. Third, we have only crudely adjusted for patient case mix in our analysis. Changes in case mix might have occurred over the study period due reductions in bed numbers, rises in involuntary admissions, ${ }^{13}$ and the widespread introduction of alternatives to in-patient admission such as crisis resolution and home treatment teams. These changes might have led to an increase in the overall morbidity over time of those who are admitted to hospital. However, in secondary analyses crudely controlling for psychiatric diagnoses our findings were largely unchanged, although we were unable to adjust for markers of severity. Fourth, our analysis of non-fatal self-harm is restricted to episodes requiring hospital admission - such cases represent only half of all hospital-presenting cases ${ }^{14}$ and an even smaller proportion of all episodes of self-harm. Nevertheless, those admitted to hospital are likely to be the most medically serious cases, more similar in their characteristics to those who died by suicide. Fifth, other policy measures, such as the National Institute for Health and Clinical Excellence (NICE) 2004 guidelines on the management and secondary prevention of self-harm, ${ }^{15}$ may have resulted in changes in hospital admission policies for self-harm, and these rather than other initiatives might underlie the observed reductions in self-harm admission. Lastly, this is an ecological analysis, and temporal association does not imply causation. Randomised trials or comparison of our findings with temporal trends in the timing of suicidal behaviour in other countries that have not implemented changes in post-discharge aftercare such as those in the UK might increase confidence in our findings.

\section{Findings in the context of the literature}

The heightened risk of suicide in the early post-discharge period has been reported in studies from a range of different countries with different levels of service provision. ${ }^{1,4-6}$ However, to the best of our knowledge there has been no previous evaluation of the impact of policies designed to reduce this risk. A randomised controlled trial in the USA in the 1970s evaluated the impact on suicide risk of regular letters of support sent by a psychiatrist over a 5-year period to former patients who did not wish to be followed up. ${ }^{16}$ The intervention resulted in a reduction in suicide rates over the intervention period. Studies investigating risk factors for post-discharge suicide have shown that a number of clinical and care factors are associated with increased risk (such as gender, diagnosis, a history of previous suicidal behaviour, patientinitiated discharge, missed last contact, length of stay and psychiatrist characteristics). ${ }^{17-19}$

Previous studies have shown that rates of suicide in the early and late post-discharge periods fluctuate over time. ${ }^{3,20}$ For example, a study based on linked data from Scotland showed that between 1980 and 1995 - a period of change in the characteristics of psychiatric service provision - there were increases in female suicide but declines in male suicide in the 28 days following hospital discharge. ${ }^{3}$ Such studies highlight the challenge of studying the impact of new interventions using time series approaches without an adequate control population.

\section{Implications of the study}

Our study highlights the heightened risk of fatal and non-fatal self-harm in the first few weeks after hospital discharge - around a quarter of suicides and hospital admissions for self-harm in the 12 months following discharge occurred within 4 weeks of discharge. We have found some evidence that recent policy initiatives to reduce the risk of suicide in this period have had a beneficial impact on the risk of self-harm. The Department of Health has recently strengthened its guidance for high-risk patients. It has recommended that they are supported by telephone contacts with ward staff or an identified alternative when on leave or discharged and should also have a 'return to the ward' plan in place. It is therefore possible that beneficial impacts of current policy may increase. ${ }^{21}$

Our study also highlights the difficulties inherent in evaluating policy interventions. At a time when NHS funds are coming under increasing pressure, evidence is essential to support continuation of new activity. In the case of new service developments which are introduced without comparison populations, potential approaches to evaluation include 'before and after' studies. The National Confidential Inquiry is embarking on just such work. ${ }^{22}$ Further research should investigate the impact of case-mix changes on the observed patterns of risk and use gold standard research designs, such as randomised trials or randomised rollout of new interventions using stepped wedge designs, to better understand and ameliorate the risk of suicide in the early weeks following hospital discharge.

\section{David Gunnell, DSC, Chris Metcalfe, PhD, David While, PhD, Keith Hawton, DSC, Davidson Ho, MSC, Louis Appleby, FRCPsych, Nav Kapur, FRCPsych, School of Social and Community Medicine, University of Bristol; Centre for Suicide Prevention, Centre for Mental Health and Risk, University of Manchester; Centre for Suicide Research, University of Oxford Department of Psychiatry, Oxford, UK}

Correspondence: David Gunnell, School of Social and Community Medicine, 39 Whatley Road, University of Bristol, Bristol BS8 2PS, UK. Email:

d.j.gunnell@bristol.ac.uk

First received 10 Oct 2011, final revision 27 Feb 2012, accepted 15 Mar 2012

\section{Funding}

This article presents independent research commissioned by the National Institute for Health Research under its Programme Grants for Applied Research scheme (RP-PG-06061247). The National Confidential Inquiry into Suicide and Homicide by People with Mental Illness was funded by the National Patient Safety Agency and is currently funded by the Health Quality Improvement Partnership.

\section{Acknowledgements}

Hospital Episode Statistics were provided by the National Health Service Information Centre for Health and Social Care. We acknowledge the staff at the National Confidential Inquiry into Suicide and Homicide by People with Mental IIIness for their role in the collection and processing of the suicide data.

\section{References}

1 Goldacre M, Seagroatt V, Hawton K. Suicide after discharge from psychiatric inpatient care [see comments]. Lancet 1993; 342: 283-6.

2 Meehan J, Kapur N, Hunt IM, Turnbull P, Robinson J, Bickley H, et al. Suicide in mental health in-patients and within 3 months of discharge. National clinical survey. Br J Psychiatry 2006; 188: 129-34. 
3 Geddes JR, Juszczak E. Period trends in rate of suicide in first 28 days after discharge from psychiatric hospital in Scotland, 1968-92. BMJ 1995; 311: 357-60.

4 Qin $\mathrm{P}$, Nordentoft M. Suicide risk in relation to psychiatric hospitalization: evidence based on longitudinal registers. Arch Gen Psychiatry 2005; 62: 427-32

5 Thong JY, Su AH, Chan $\mathrm{YH}$, Chia BH. Suicide in psychiatric patients: case-control study in Singapore. Aust N Z J Psychiatry 2008; 42: 509-19.

6 Ho T-P. The suicide risk of discharged psychiatric patients. J Clin Psychiatry 2003; 64: 702-7.

7 Gunnell D, Hawton K, Ho D, Evans J, O'Connor S, Potakar J, et al. Hospital admissions for self-harm after discharge from psychiatric inpatient care: cohort study. BMJ 2008; 337: a2278.

8 Department of Health. National Suicide Prevention Strategy for England. Department of Health, 2002.

9 National Institute for Mental Health in England. Preventing Suicide: A Toolkit for Mental Health Services. NIMHE, 2003.

10 Appleby L, Shaw J, Kapur N, Windfuhr K, Ashton A, Swinson N, et al. Avoidable Deaths: Five Year Report of the National Confidential Inquiry into suicide and Homicide by People with Mental IIIness. University of Manchester, 2006.

11 World Health Organization. International Statistical Classification of Diseases and Related Health Problems (ICD-10). WHO, 1992.

12 Sellar C, Goldacre MJ, Hawton K. Reliability of routine hospital data on poisoning as measures of deliberate self poisoning in adolescents. J Epidemiol Community Health 1990; 44: 313-5.

13 Keown P, Weich S, Bhui KS, Scott J. Association between provision of mental illness beds and rate of involuntary admissions in the NHS in England 19882008: ecological study BMJ 2011; 343: d3736.
14 Gunnell D, Bennewith O, Peters TJ, House A, Hawton K. The epidemiology and management of self-harm amongst adults in England. $J$ Public Health (Oxf) 2005; 27: 67-73.

15 National Institute for Health and Clinical Excellence. Self-harm. The Shortterm Physical and Psychological Management and Secondary Prevention of Self-harm in Primary and Secondary Care. (Clinical Guideline 16). NICE, 2004 (http://www.nice.org.uk/nicemedia/pdf/CG016NICEguideline.pdf).

16 Motto JA, Bostrom AG. A randomized controlled trial of post-crisis suicide prevention. Psychiatr Serv 2001; 52: 828-33.

17 Hunt IM, Kapur N, Webb R, Robinson J, Burns J, Shaw J, et al. Suicide in recently discharged psychiatric patients: a case-control study. Psychol Med 2009; 39: 443-9.

18 Lee $\mathrm{HC}$, Lin $\mathrm{HC}$. Are psychiatrist characteristics associated with postdischarge suicide of schizophrenia patients? Schizophr Bull 2009; 35: 760-5.

19 King EA, Baldwin DS, Sinclair JMA, Baker NG, Campbell M, Thompson C. The Wessex Recent In-Patient Suicide Study, 1. Case-control study of 234 recently discharged psychiatric patient suicides. Br J Psychiatry 2001; 178 $531-6$.

20 Lawrence DM, Holman CD, Jablensky AV, Fuller SA. Suicide rates in psychiatric in-patients: an application of record linkage to mental health research. Aust N Z J Publ Health 1999; 23: 468-70.

21 National Patient Safety Agency. Preventing Suicide: A Toolkit for Mental Health Services. NPSA, 2009

22 While D, Bickley $H$, Roscoe A, Windfuhr $K$, Rahman S, Shaw J, et al. Implementation of mental health service recommendations in England and Wales and suicide rates, 1997-2006: a cross-sectional and before-and-after observational study. Lancet 2012; 379: 1005-12. 